\title{
Water-based superlubricity in vacuum
}

\author{
Chen XIAO ${ }^{1}$, Jinjin LI $^{2}$, Lei CHEN ${ }^{1}$, Chenhui ZHANG ${ }^{2}$, Ningning ZHOU ${ }^{3}$, Tao QING ${ }^{3}$, Linmao QIAN ${ }^{1, *}$, \\ Jiyang ZHANG ${ }^{3}$, Jianbin LUO ${ }^{2}$ \\ ${ }^{1}$ Tribology Research Institute, State Key Laboratory of Traction Power, Southwest Jiaotong University, Chengdu 610031, China \\ ${ }^{2}$ State Key Laboratory of Tribology, Tsinghua University, Beijing 100084, China \\ ${ }^{3}$ Beijing Key Laboratory of Long-life Technology of Precise Rotation and Transmission Mechanisms, Beijing Institute of Control Engineering, \\ Beijing 100094, China
}

Received: 10 September 2017 / Revised: 06 November 2017 / Accepted: 10 February 2018

(C) The author(s) 2018. This article is published with open access at Springerlink.com

\begin{abstract}
This study achieved water-based superlubricity with the lubrication of $\mathrm{H}_{3} \mathrm{PO}_{4}$ solution in vacuum (highest vacuum degree $<10^{-4}$ torr) for the first time by performing a pre-running process in air before running in vacuum. The stable water-based superlubricity was sustainable in vacuum ( 0.02 torr) for $14 \mathrm{~h}$ until the test was stopped by the user for non-experimental factor. A further analysis suggested that the superlubricity may be attributed to the phosphoric acid-water network formed in air, which can efficiently lock water molecules in the liquid lubricating film even in vacuum owing to the strong hydrogen bond interaction. Such capability to lock water is strongly affected by the strength of hydrogen bond and environmental conditions. The realization of water-based superlubricity with $\mathrm{H}_{3} \mathrm{PO}_{4}$ solution in vacuum can lead to its application in space environment.
\end{abstract}

Keywords: friction; water-based superlubricity; vacuum; phosphoric acid

\section{Introduction}

Due to their low mechanical noise, high lubricating stability, self-restoration capability, and insensitivity to environmental factors, liquid lubricants have been widely used to meet the long-term reliable and accurate operational requirements of space mechanisms [1-4]. Liquid lubricants can be divided into two types, i.e., oil-based and water-based. Given that rapid water evaporation occurs under low-pressure condition in space, water-based lubricants have not been used in space applications [4,5]. However, water-based lubricants are one of the most promising lubricants because they are cost-effective, highly reproducible, and environmentally friendly [6]. In addition, waterbased lubricating films have excellent fluidity even under high contact pressures and can usually generate friction-induced tribochemical reactions with tribopair surfaces, which creates favorable conditions for realizing superlubricity [7-9]. Superlubricity, in which the friction coefficient between two contacting surfaces is less than 0.01 , presents a unique advantage for saving energy, improving operation reliability, and increasing the working life of space mechanisms [10-13].

Previous studies have proposed different types of water-based superlubricants, such as ceramic materials with water, polymer brushes, glycerol solution with acid or polyhydric alcohol, and polysaccharide mucilage from plants [14-18]. The superlubricity of these systems is mainly attributed to the formation of a hydrated water layer between the tribopair surfaces with extremely low shear strength [19-21]. Chen et al. reported that environmental humidity has a significant effect on the lubrication behaviors of water-based superlubricants [22]. The experimental results showed that the water content in the liquid lubricating film played a key role in the superlubricity behavior. When the humidity is low, the water evaporation rate of the

* Corresponding author: Linmao QIAN, E-mail: linmao@swjtu.edu.cn 
liquid lubricating film is higher than that of water absorption, which destroys the hydrated water layer and results in superlubricity failure. Therefore, in view of the severe low-pressure environment in space, strict control of the water content in liquid lubricating film has become an important method to achieve waterbased superlubricity. To the best of our knowledge, until recently, no available information regarding the water-based superlubricity achieved in vacuum can be found in the previous literature. Thus, it is necessary to carry out the research on water-based superlubricity in vacuum to satisfy the increasing demands for high reliability and long-term requirements of space lubrication.

In the present work, the effect of vacuum environment on the lubrication behavior with $\mathrm{H}_{3} \mathrm{PO}_{4}$ solution was investigated. After the phosphoric acidwater network was formed by performing the prerunning-in process in air, we found that the stable water-based superlubricity was sustainable in vacuum ( 0.02 torr) for $14 \mathrm{~h}$ until the test was stopped by user for non-experimental factor. The analysis suggested that the stable vacuum-superlubricity state may be attributed to the strong hydrogen bond interaction in the phosphoric acid-water network formed in air, which can efficiently lock water molecules in the liquid lubricating film. The results creatively overcome the difficulty in maintaining the water molecules in the liquid lubricating film in vacuum and provide new insights into the applications of water-based superlubricity in space.

\section{Materials and methods}

The liquid lubricant used in all friction tests was $\mathrm{H}_{3} \mathrm{PO}_{4}$ solution with a $\mathrm{pH}$ of 1.5 , which is measured by a standard $\mathrm{pH}$ meter after pre-calibration. It was diluted by a commercial product (purity greater than 99\%) purchased from Sinopharm Chemical Reagent Co. Ltd. with a concentration of $85 \mathrm{wt}$.\%. The tribopair consisted of $\mathrm{Si}_{3} \mathrm{~N}_{4}$ ball with a diameter of $4 \mathrm{~mm}$ and glass slide with a root mean square (RMS) roughness of approximately $0.5 \mathrm{~nm}$ over a $400 \mu \mathrm{m}^{2}$ area. Before the friction tests, both the $\mathrm{Si}_{3} \mathrm{~N}_{4}$ ball and glass slide were ultrasonically cleaned in acetone, ethanol, and deionized water for $10 \mathrm{~min}$ in sequence to remove surface contamination, and then dried by pure nitrogen gas. All the friction tests were performed using a universal micro-tribotester (UMT-V, Bruker, USA) with ball-on-disk mode, as illustrated in the inset of Fig. 1(a). When the tests were conducted in air, the relative humidity was controlled at $50 \pm 1 \%$. To simulate the actual working environment of bearings that work inside the aircraft, the vacuum tests were performed in a vacuum chamber and the degree of vacuum was set at $10^{-2}$ torr [23]. A drop $(10 \mu \mathrm{L})$ of liquid lubricant was added between the $\mathrm{Si}_{3} \mathrm{~N}_{4}$ ball and glass substrate before test. During the whole tests, the applied normal load was $3 \mathrm{~N}$, which provided a maximum contact pressure of $830 \mathrm{MPa}$ based on the Hertzian contact theory [24], the liner speed was $0.2 \mathrm{~m} / \mathrm{s}$, and the temperature was controlled at $25 \pm 2{ }^{\circ} \mathrm{C}$. In order to ensure the ultra-high measurement accuracy of friction, the measuring error could be reduced significantly by the pre-commission process. More details regarding this can be found in the previous literature [25].

\section{Results and discussions}

\subsection{Water-based superlubricity achieved in vacuum}

In order to investigate the effect of vacuum on the superlubricity behavior of $\mathrm{Si}_{3} \mathrm{~N}_{4}$ /glass pair with $\mathrm{H}_{3} \mathrm{PO}_{4}$ solution, friction tests were performed in vacuum and the results are shown in Fig. 1(a). The friction test was divided into two steps: (I) achieve superlubricity state after the running-in process in air, and then suspend the experiment temporarily; (II) restart the test after the chamber is evacuated (0.02 torr). As shown in Fig. 1(a), the friction coefficient decreases to 0.006 after the running-in period of $200 \mathrm{~s}$ in air and then it stabilizes. Moreover, after restarting the test in vacuum, the friction coefficient further decreases to 0.003 without running-in process and the stable superlubricity state can be sustained for at least $14 \mathrm{~h}$ (including $9 \mathrm{~h}$ of standby time). Finally, the test was stopped by the user for non-experimental factor, and the superlubricity system was still effective at the end of test. Notably, the friction coefficient of the superlubricity state achieved in vacuum environment was lower than that in the atmosphere. Moreover, the superlubricity state is extremely stable in running and suspended stages. The morphologies of the wear 
(a)
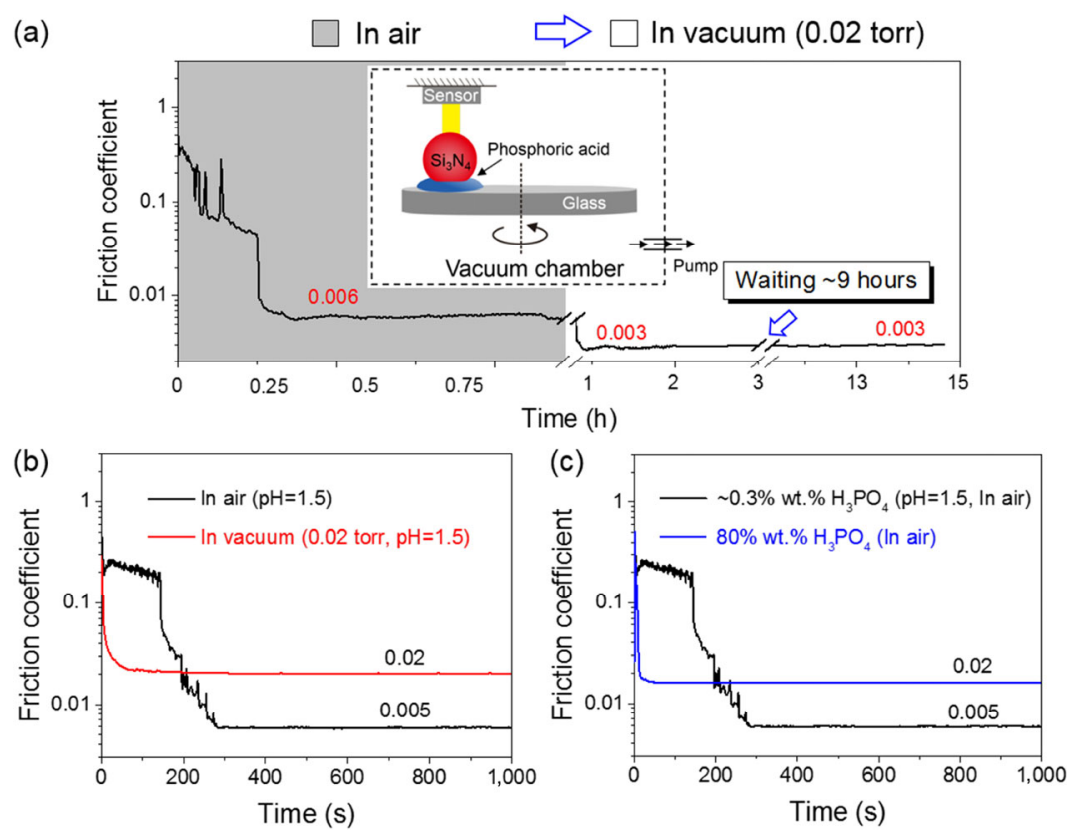

Fig. 1 (a) Liquid superlubricity system with $\mathrm{H}_{3} \mathrm{PO}_{4}$ solution remains effective in vacuum for $14 \mathrm{~h}$ (including $9 \mathrm{~h}$ of standby time) after the pre-running-in process in air. The inset shows the schematic illustration of friction measurement system with ball-on-disk mode. (b) Friction coefficient measured in vacuum without pre-running-in process in air. The result of test performed in air is also plotted as a comparison. (c) Effect of water content in liquid lubricating film on the lubrication behavior of $\mathrm{H}_{3} \mathrm{PO}_{4}$ solution.

tracks on the tribopair surfaces in vacuum and ambient environments are shown in Fig. S1 in the Electronic Supplementary Material (ESM). There is little difference in the wear depth of wear tracks on tribopair surfaces, which indicates that the vacuum environment has little effect on the wear behavior between the $\mathrm{Si}_{3} \mathrm{~N}_{4}$ balls and glass substrates. Furthermore, a wear test was conducted under higher vacuum degree $\left(<10^{-4}\right.$ torr $)$, and the superlubricity state could also be maintained, as shown in Fig. 2. Similar to the results obtained at 0.02 torr, the friction coefficient further decreased from 0.007 to 0.0035 in the vacuum. Previous studies pointed

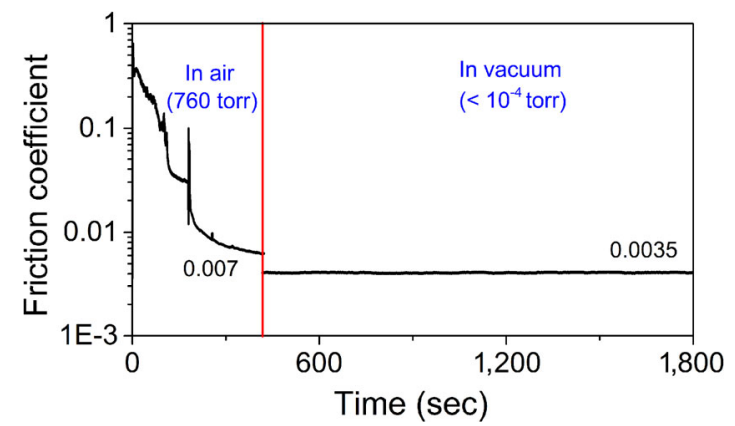

Fig. 2 Superlubricity state with $\mathrm{H}_{3} \mathrm{PO}_{4}$ solution maintains in higher vacuum degree condition ( $<10^{-4}$ torr) after the pre-running-in process in air. out that the superlubricity mechanism in air may be attributed to the hydrated water layer with hydrogen bond network among $\mathrm{H}_{3} \mathrm{PO}_{4}, \mathrm{H}_{2} \mathrm{PO}_{4}^{-}$, and $\mathrm{H}_{2} \mathrm{O}$ [19]. The results suggest that the phosphoric acid-water network formed in air can be barely destroyed and it even presents a better superlubricity performance in vacuum, which may be attributed to the more compact hydrogen bond network induced by structural transformations in vacuum [26].

However, when the test was performed in vacuum without the pre-running-in process in air, the superlubricity state cannot be achieved until the end of test, and the friction coefficient can just enter traditional hydrodynamic lubrication state $(\mu=0.02)$, as shown in Fig. 1(b). Superlubricity failure in vacuum may be attributed to the accelerated water evaporation. Thus, the friction tests under the lubrication of $\mathrm{H}_{3} \mathrm{PO}_{4}$ solution with different concentrations were performed in air to verify the aforementioned process, as shown in Fig. 1(c). The evolution of friction coefficient in vacuum is similar to that under the lubrication of $80 \mathrm{wt} . \% \mathrm{H}_{3} \mathrm{PO}_{4}$ solution in air, which is also consistent with the results by Li et al. [27]. Above results reveal that the pre-running-in process in air plays an 
important role in the subsequent vacuum-superlubricity, and the main reason for superlubricity failure in vacuum may be similar to that under the lubrication of $\mathrm{H}_{3} \mathrm{PO}_{4}$ solution with high concentration. A free water layer is necessary because it provides a low shearing strength to lower the friction force, which is the origin of superlubricity [19]. However, too much free water will restrain the formation of starvation state and hydrogen bond network [28]. It can be speculated that the loss of free water in lubricating film caused by rapid water evaporation leads to an insufficient running-in process, as well as lack of time for the tribochemical reaction to occur, thereby resulting in the failure of vacuum-superlubricity [27]. Therefore, stable superlubricity is unachievable when the test is performed in vacuum without prerunning-in process in air, but it can be realized after the formation of phosphoric acid-water network in air. This work introduces new insights into achieving water-based superlubricity in vacuum, which provides the possibility for the application of water-based superlubricants in space.

\subsection{Mechanism of water-based superlubricity achieved in vacuum}

A large number of studies have shown that there is a complex interaction between phosphate groups and water molecules $[29,30]$. Since $\mathrm{H}_{3} \mathrm{PO}_{4}$ molecules contain high-density $\mathrm{P}=\mathrm{O}$ bonds and $\mathrm{OH}$ groups, the hydrogen bond interaction between $\mathrm{H}_{3} \mathrm{PO}_{4}$ and water molecules can be formed in various combinations according to the formation conditions of hydrogen bonds [31]. Moreover, $\mathrm{H}_{3} \mathrm{PO}_{4}$ and water molecules can also form a strong hydrogen bond network with each other. In many stable configurations, $\mathrm{H}_{3} \mathrm{PO}_{4}$ and water molecules are not only the providers of proton but also their acceptors [32]. Thus, they tend to form a multi-cyclic structure with abundant hydrogen bonds and reduce the distance between the molecules [33]. This synergistic effect results in a considerable stability of the hydrogen bond network, which is also conducive to develop a strong hydrogen bond interaction between $\mathrm{H}_{3} \mathrm{PO}_{4}$ and water molecule. Figure 3 shows the possible molecular model of phosphoric acid-water network, more details about the tribochemical reaction processes can be found in the previous literatures $[19,22,26,28,34]$. For the vast majority of liquid lubricants in vacuum, water molecules in lubricating film would rapidly evaporate, but no water can be absorbed back. However, the phenomenological results show that, even in vacuum environment, the phosphoric acid-water layer with strong hydrogen bond interaction may efficiently lock the water molecules in the lubricating film and further prevent superlubricity failure.

In order to understand the influence of hydrogen bond strength in hydrated water layer on water-based superlubricity behavior in vacuum, the same experiments were performed with the lubrication of mixture of glycerol $\left(\mathrm{C}_{3} \mathrm{H}_{8} \mathrm{O}_{3}\right)$ and $\mathrm{H}_{3} \mathrm{PO}_{4}$ solutions, which was obtained by mixing the $\mathrm{H}_{3} \mathrm{PO}_{4}$ solution with $\mathrm{C}_{3} \mathrm{H}_{8} \mathrm{O}_{3}$ solution $(20 \% \mathrm{v} / \mathrm{v})$ and the $\mathrm{pH}$ value was controlled at approximately 1.5. In addition to the liquid lubricant, other experimental parameters kept the same as mentioned above. Figure 4 shows that the superlubricity state is achieved after the running-in process in air, which is attributed to the hydrogen bond network formed by $\mathrm{C}_{3} \mathrm{H}_{8} \mathrm{O}_{3}$ and water molecules with low shear strength [35]. However, the friction coefficient immediately increases from 0.007 to 0.03 when the

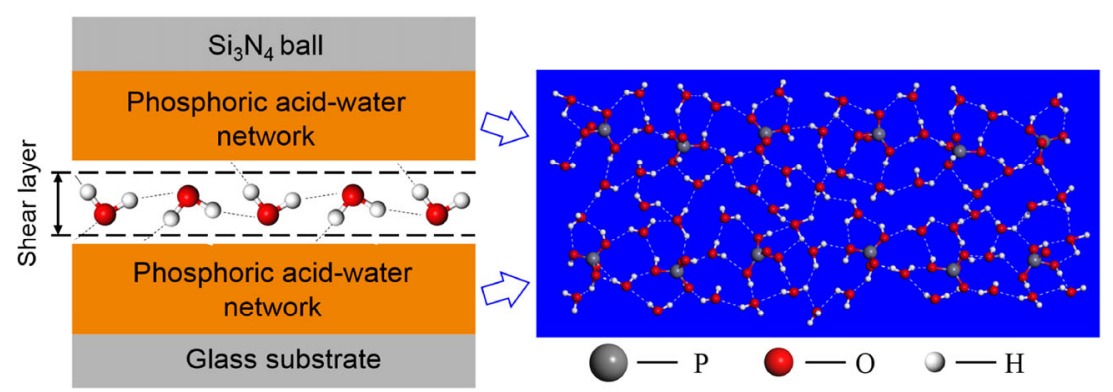

Fig. 3 Schematic illustration of the liquid lubricating film between two friction surfaces (left) and the possible molecular schematic representation of phosphoric acid-water network (right) [19]. 


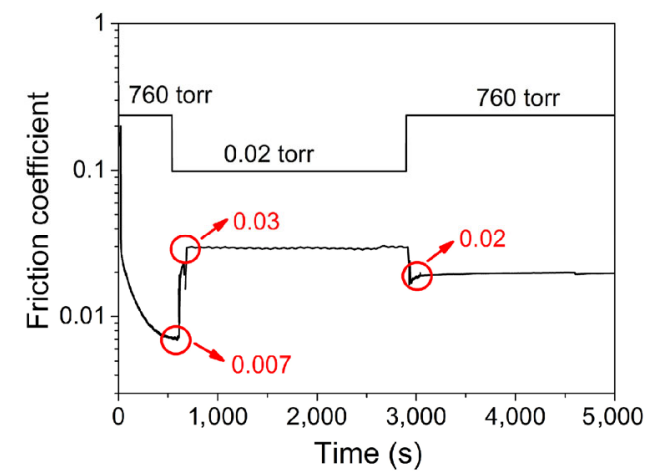

Fig. 4 Effect of the hydrogen bond strength in hydrated water layer on water-based superlubricity behavior in vacuum. The friction coefficient is measured under the lubrication of mixture of glycerol and $\mathrm{H}_{3} \mathrm{PO}_{4}$ solutions, and the other experimental parameters are the same as those in Fig. 1.

chamber pressure is pumped to 0.02 torr. After the experimental environment is returned to the atmosphere condition, some free water in air may come into the lubricant and induce a little reduction in friction coefficient but the superlubricity state is still not restored. We also repeated that experiment with the lubrication of mixture of glycerol and $\mathrm{H}_{2} \mathrm{SO}_{4}$ solutions, whose $\mathrm{pH}$ value was also controlled as 1.5, as shown in Fig. S2 in the ESM. The results show that the effect of the type of acids on superlubricity failure in vacuum can be ignored. The above results indicate that the glycerol-water network cannot effectively prevent water loss in the liquid lubricating film under low-pressure condition, which results in irreversible superlubricity failure. Since the $\mathrm{H}_{3} \mathrm{PO}_{4}$ molecule has a higher density of hydroxyl or carboxyl groups (approximately $74 \mathrm{~mol} / \mathrm{L}$ for $94 \mathrm{wt} . \%$ solution) than $\mathrm{C}_{3} \mathrm{H}_{8} \mathrm{O}_{3}$ molecule (approximately $41 \mathrm{~mol} / \mathrm{L}$ for $92 \mathrm{wt} . \%$ solution). Therefore, the tetrahedral structure $\left(\mathrm{H}_{3} \mathrm{PO}_{4}\right)$ could form a more compact molecular configuration with water molecules compared with the chain structure $\left(\mathrm{C}_{3} \mathrm{H}_{8} \mathrm{O}_{3}\right)$, and the hydrogen bond strength in phosphoric acid-water network is much stronger than that in glycerol-water network [33]. Thus, the superlubricity state in vacuum may be attributed to the phosphoric-water network formed in the prerunning-in process which could efficiently lock the water molecules in the lubricating film. Moreover, the capability of lubricant molecules to lock water is strongly affected by the hydrogen bond strength and environmental conditions (vacuum degree). According to our results, strong hydrogen bond network is conducive in achieving a stable liquid superlubricity in vacuum. Therefore, the molecular structure with high-density functional groups, which can form compact hydrogen bond networks (such as hydroxyl, carboxyl, and amidogen groups) should be considered in the future design of water-based superlubricity in vacuum. The results present in this paper may open a door to the liquid superlubricity in vacuum environment.

\section{Conclusions}

This work demonstrates water-based superlubricity in vacuum (highest vacuum degree $<10^{-4}$ torr) for the first time. After the phosphoric acid-water network was formed by performing the pre-running-in process in air, the stable water-based superlubricity state was sustainable in vacuum for at least $14 \mathrm{~h}$. The phosphoric acid-water network may hold the water molecules in vacuum by the strong hydrogen bond interaction. Moreover, the capability to lock water is strongly affected by the hydrogen bond strength and environmental conditions.

\section{Acknowledgements}

The authors are grateful for the financial support from the National Natural Science Foundation of China (Nos. 51527901, 51405256), and Self-developed Project of State Key Laboratory of Traction Power (No. 2017TPL_Z02).

Electronic Supplementary Material: Supplementary material is available in the online version of this article at https://doi.org/10.1007/s40544-018-0212-z.

Open Access: The articles published in this journal are distributed under the terms of the Creative Commons Attribution 4.0 International License (http:// creativecommons.org/licenses/by/4.0/), which permits unrestricted use, distribution, and reproduction in any medium, provided you give appropriate credit to the original author(s) and the source, provide a link to the Creative Commons license, and indicate if changes were made. 


\section{References}

[1] Fan X Q, Xue Q J, Wang L P. Carbon-based solid-liquid lubricating coatings for space applications-a review. Friction 3(3): 191-207 (2015)

[2] Jones Jr W R, Shogrin B A, Jansen M J. Research on liquid lubricants for space mechanisms. Lubr Sci 17(2): 109-122 (2000)

[3] Masuko M, Jones Jr W R, Helmick L S. Tribological characteristics of perfluoropolyether liquid lubricants under sliding conditions in high vacuum. Lubr Sci 11(2): 111-119 (1994)

[4] Zaretsky E V. Liquid lubrication in space. Tribol Int 23(2): 75-93 (1990)

[5] Bhushan B. Principles and Applications of Tribology. New York (USA): John Wiley \& Sons, 2013.

[6] Xiao C, Li J J, Chen L, Zhang C H, Zhou N N, Qian L M, Luo J B. Speed dependence of liquid superlubricity stability with $\mathrm{H}_{3} \mathrm{PO}_{4}$ solution. RSC Adv 7(78): 49337-49343 (2017)

[7] Li J J, Zhang C H, Deng M M, Luo J B. Investigation of the difference in liquid superlubricity between water- and oil-based lubricants. RSC Adv 5(78): 63827-63833 (2015)

[8] Li J J, Luo J B. Advancements in superlubricity. Sci China Technol Sci 56(12): 2877-2887 (2013)

[9] Raviv U, Laurat P, Klein J. Fluidity of water confined to subnanometre films. Nature 413(6851): 51-54 (2001)

[10] Hirano M, Shinjo K. Atomistic locking and friction. Phys Rev B Condens Matter 41(17): 11837-11851 (1990)

[11] Shinjo K, Hirano M. Dynamics of friction: superlubric state. Surf Sci 283(1-3): 473-478 (1993)

[12] Erdemir A, Martin J M. Superlubricity. New York (USA): Elsevier, 2007.

[13] $\mathrm{Xu} \mathrm{J,} \mathrm{Li} \mathrm{J} \mathrm{J.} \mathrm{New} \mathrm{achievements} \mathrm{in} \mathrm{superlubricity} \mathrm{from}$ international workshop on superlubricity: fundamental and applications. Friction 3(4): 344-351 (2015)

[14] Liu P X, Liu Y H, Yang Y Y, Chen Z, Li J J, Luo J B. Mechanism of biological liquid superlubricity of Brasenia schreberi mucilage. Langmuir 30(13): 3811-3816 (2014)

[15] Li J J, Zhang C H, Deng M M, Luo J B. Superlubricity of silicone oil achieved between two surfaces by running-in with acid solution. RSC Adv 5(39): 30861-30868 (2015)

[16] Chen M, Briscoe W H, Armes S P, Klein J. Lubrication at physiological pressures by polyzwitterionic brushes. Science 323(5922): 1698-1701 (2009)

[17] Klein J, Kumacheva E, Mahalu D, Perahia D, Fetters L J. Reduction of frictional forces between solid surfaces bearing polymer brushes. Nature 370(6491): 634-636 (1994)

[18] Chen M, Kato K, Adachi K. Friction and wear of self-mated $\mathrm{SiC}$ and $\mathrm{Si}_{3} \mathrm{~N}_{4}$ sliding in water. Wear 250(1-12): 246-255
(2001)

[19] Li J J, Zhang C H, Luo J B. Superlubricity behavior with phosphoric acid-water network induced by rubbing. Langmuir 27(15): 9413-9417 (2011)

[20] Klein J. Hydration lubrication. Friction 1(1): 1-23 (2013)

[21] Deng M M, Zhang C H, Li J J, Ma L R, Luo J B. Hydrodynamic effect on the superlubricity of phosphoric acid between ceramic and sapphire. Friction 2(2): 173-181 (2014)

[22] Chen Z, Liu Y H, Zhang S H, Luo J B. Controllable superlubricity of glycerol solution via environment humidity. Langmuir 29(38): 11924-11930 (2013)

[23] Kameno H, Kubo A, GÄCHTER S, Takahata R. Basic design of $1 \mathrm{kWh}$ class compact flywheel energy storage system-application of active magnetic bearings with zeropower nonlinear control method. Koyo Eng J Engl Edit 163E: $44-48$ (2003)

[24] Johnson K L. Contact Mechanics. Cambridge (UK): Cambridge University Press, 1987.

[25] Li J J, Zhang C H, Sun L, Luo J B. Analysis of measurement inaccuracy in superlubricity Tests. Tribol Trans 56(1): 141-147 (2013)

[26] Zhou G P, Wang Y B. Density function theory on intermolecular interactions of $\mathrm{H}_{3} \mathrm{PO}_{4}$ with $\mathrm{H}_{2} \mathrm{O}$. J Northwest Norm Univ Nat Sci 48(1): 74-79 (2012)

[27] Li J J, Zhang C H, Luo J B. Effect of $\mathrm{pH}$ on the liquid superlubricity between $\mathrm{Si}_{3} \mathrm{~N}_{4}$ and glass achieved with phosphoric acid. $R S C A d v$ 4(86): 45735-45741 (2014)

[28] Li J J, Zhang C H, Deng M M, Luo J B. Investigations of the superlubricity of sapphire against ruby under phosphoric acid lubrication. Friction 2(2): 164-172 (2014)

[29] Pihko P M. Hydrogen Bonding in Organic Synthesis. Weinheim (Germany): Wiley-VCH, 2009.

[30] Jeffrey G A, Saenger W. Hydrogen Bonding in Biological Structures. Berlin (Germany): Springer, 2012.

[31] Gilmour R. Phosphoric Acid: Purification, Uses, Technology, and Economics. Boca Raton, Florida (USA): CRC Press, 2013.

[32] Israelachvili J N. Intermolecular and Surface Forces. Pittsburgh (USA): Academic Press, 2011.

[33] Ewig C S, Van Wazer J R. Ab initio structures of phosphorus acids and esters. 1. Phosphinic, phosphonic, and phosphoric acids. J Am Chem Soc 107(7): 1965-1971 (1985)

[34] Li J J, Ma L R, Zhang S H, Zhang C H, Liu Y H, Luo J B. Investigations on the mechanism of superlubricity achieved with phosphoric acid solution by direct observation. $J$ Appl Phys 114(11): 114901 (2013)

[35] Li J J, Zhang C H, Ma L R, Liu Y H, Luo J B. Superlubricity achieved with mixtures of acids and glycerol. Langmuir 29(1): 271-275 (2013) 


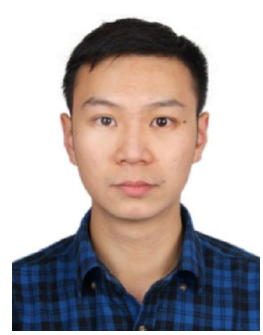

Chen XIAO. He earned his B.E. degree (2014) in mechanical design, manufacturing and its automation from Southwest Jiaotong University in China. He is currently a $\mathrm{PhD}$

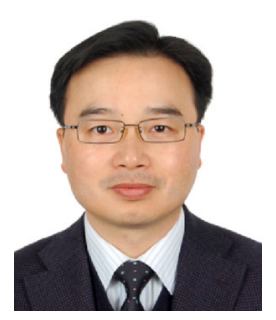

Linmao QIAN. He is a professor of mechanical engineering at Southwest Jiaotong University. He received his B.S. (1994) and $\mathrm{PhD}$ (2000) degrees in mechanical engineering from Tsinghua University in China. After two years at Ecole Normale Supérieure in Paris and one year at Hong Kong University of Science and Technology as a postdoctoral researcher, he joined the faculty at Southwest candidate in Tribology Research Institute, Southwest Jiaotong University. His research is focused on the tribochemical removal on silicon and water-based superlubricity.

Jiaotong University in 2002. His research interest includes nanotribology, nanofabrication and superlubricity. He has published more than 136 peerreviewed journals papers and authorized 18 patents. He is a member of editorial board of six journals, such as Friction, ISRN Tribology, Frontiers of Mechanical Engineering, and the Proceedings of the Institution of Mechanical Engineers, Part J: Journal of Engineering Tribology. He can be reached at linmao@swjtu.edu.cn. 